\title{
Poisson Equation in the Kohn-Sham Coulomb Problem
}

\author{
F. R. Manby* and P. J. Knowles ${ }^{\dagger}$ \\ School of Chemistry, University of Birmingham, Edgbaston, Birmingham B15 2TT, United Kingdom
}

(Received 16 March 2001; published 27 September 2001)

\begin{abstract}
We apply the Poisson equation to the quantum mechanical Coulomb problem for many-particle systems. By introducing a suitable basis set, the two-electron Coulomb integrals become simple overlaps. This offers the possibility of very rapid linear-scaling treatment of the Coulomb contribution to KohnSham theory.
\end{abstract}

DOI: 10.1103/PhysRevLett.87.163001

Consider a charge density $\rho(\mathbf{r})$ describing an $N$-particle system. The classical Coulomb energy is given by

$$
E_{J}[\rho]=\frac{1}{2} \int d \mathbf{r}_{1} \int d \mathbf{r}_{2} \frac{\rho\left(\mathbf{r}_{1}\right) \rho\left(\mathbf{r}_{2}\right)}{\left|\mathbf{r}_{1}-\mathbf{r}_{2}\right|},
$$

and the cost of evaluating it scales formally quadratically with $N$. This $\mathcal{O}\left(N^{2}\right)$ scaling makes the Coulomb evaluation a major bottleneck in Kohn-Sham theory [1] applied to systems of many electrons. In such theories $\rho$ is expanded in a set of functions (which may be of direct product form)

$$
\rho(\mathbf{r})=\sum_{i} d_{i} \chi_{i}(\mathbf{r}),
$$

and the Coulomb energy is then written (with summation over repeated indices) $E_{J}=\frac{1}{2} d_{i} J_{i j} d_{j}$, where

$$
J_{i j}=\int d \mathbf{r}_{1} \int d \mathbf{r}_{2} \frac{\chi_{i}\left(\mathbf{r}_{1}\right) \chi_{j}\left(\mathbf{r}_{2}\right)}{\left|\mathbf{r}_{1}-\mathbf{r}_{2}\right|} .
$$

Linear scaling can be achieved by suitable partitioning of the Coulomb interaction into short- and long-ranged parts and continuous fast multipole methods [2-6]. Even though the number of short-range Coulomb integrals scales linearly in such methods, each integral is inherently rather time consuming to compute, despite huge advances in integral evaluation technology. In this Letter we present a linear-scaling method for evaluating $E_{J}$ in which the Coulomb integrals become exceptionally simple.

Theory.-Coulomb fitting methods have seen considerable success in speeding up the evaluation of the Coulomb energy $[7,8]$. The idea is to form a model density $\tilde{\rho}(\mathbf{r})$,

$$
\tilde{\rho}(\mathbf{r})=\sum_{a} c_{a} \xi_{a}(\mathbf{r}),
$$

with $\left\{\xi_{a}\right\}$ a smaller basis than $\left\{\chi_{i}\right\}$, and then to minimize the error

$$
\Delta=\frac{1}{2} \int d \mathbf{r}_{1} \int d \mathbf{r}_{2} \frac{\left[\rho\left(\mathbf{r}_{1}\right)-\tilde{\rho}\left(\mathbf{r}_{1}\right)\right]\left[\rho\left(\mathbf{r}_{2}\right)-\tilde{\rho}\left(\mathbf{r}_{2}\right)\right]}{\left|\mathbf{r}_{1}-\mathbf{r}_{2}\right|}
$$

to determine the coefficient vector $c$. This forms one of the key elements of the current approach.

A previous study pertaining to the evaluation of the Coulomb potential on an integration grid [9] revealed that explicit use of the Poisson equation does not lead to com-
PACS numbers: $31.15 .-\mathrm{p}, 02.70 .-\mathrm{c}$

petitive methods; nevertheless, it is the Poisson equation that forms the second key element of our paper. To simplify the notation we write $P=-(4 \pi)^{-1} \nabla^{2}$. The Poisson equation,

$$
P v(\mathbf{r})=\rho(\mathbf{r}),
$$

relates the density $\rho$ to the Coulomb potential,

$$
v(\mathbf{r})=\int d \mathbf{r}^{\prime} \frac{\rho\left(\mathbf{r}^{\prime}\right)}{\left|\mathbf{r}-\mathbf{r}^{\prime}\right|} .
$$

Now we choose a basis of the form $\left\{P \xi_{a}\right\}$ in which to perform our Coulomb fitting. Thus

$$
\tilde{\rho}(\mathbf{r})=\sum_{a} c_{a} P \xi_{a}(\mathbf{r}),
$$

and from Eqs. (6) and (8) we can immediately write down the Coulomb potential $\tilde{v}$ arising from the density $\tilde{\rho}$ :

$$
\tilde{\boldsymbol{v}}(\mathbf{r})=\sum_{a} c_{a} \xi_{a}(\mathbf{r}) .
$$

For a function $f(\mathbf{r})$ vanishing at large $\mathbf{r}$ faster than $r^{-1}$, the integral identity

$$
\int d \mathbf{r}^{\prime} \frac{\nabla^{2} f\left(\mathbf{r}^{\prime}\right)}{\left|\mathbf{r}-\mathbf{r}^{\prime}\right|}=-4 \pi f(\mathbf{r})
$$

holds [10]. This identity allows us to reduce (without approximation) the Coulomb matrix elements in the auxiliary basis from 6- to 3-dimensional integrals,

$$
\begin{aligned}
J_{a b} & =\int d \mathbf{r}_{1} \int d \mathbf{r}_{2} \frac{\left[P_{1} \xi_{a}\left(\mathbf{r}_{1}\right)\right]\left[P_{2} \xi_{b}\left(\mathbf{r}_{2}\right)\right]}{\left|\mathbf{r}_{1}-\mathbf{r}_{2}\right|} \\
& =\int d \mathbf{r} \xi_{a}(\mathbf{r}) P \xi_{b}(\mathbf{r}),
\end{aligned}
$$

and the mixed-basis integrals become overlaps:

$$
\begin{aligned}
J_{a i} & =\int d \mathbf{r}_{1} \int d \mathbf{r}_{2} \frac{\left[P_{1} \xi_{a}\left(\mathbf{r}_{1}\right)\right]\left[\chi_{i}\left(\mathbf{r}_{2}\right)\right]}{\left|\mathbf{r}_{1}-\mathbf{r}_{2}\right|} \\
& =\int d \mathbf{r} \xi_{a}(\mathbf{r}) \chi_{i}(\mathbf{r}) .
\end{aligned}
$$

To clarify the situation we contrast the properties of these integrals in the case of a standard basis and the basis proposed in this Letter: the $J_{a b}$ are ordinarily 2-center, 6-dimensional integrals, whose values decay only slowly (an inverse power) with the distance between the functions $\xi_{a}, \xi_{b}$, whereas now they are 3-dimensional overlap-like 
integrals, analogous in form to kinetic energy integrals; the $J_{a i}$, ordinarily 3-center, 6-dimensional Coulomb integrals, are now 3-center, 3-dimensional overlap integrals. For Gaussian functions, commonly used in quantum chemistry, both of these types of integrals can be evaluated analytically and extremely quickly [11].

We can now set up our Coulomb fitting equations. The error in Eq. (5) is minimized when $J_{a b} c_{b}=J_{a i} d_{i}$, from which the energy can easily be shown to be $\widetilde{E}_{J}=$ $\frac{1}{2} d_{i} J_{i a}\left[J^{-1}\right]_{a b} J_{b j} d_{j}$.

Analysis and discussion. - We have presented a formalism for evaluating the Coulomb energy of a charge distribution in which the Coulomb integrals simplify to 3 -dimensional overlap integrals or integrals with kernel $\nabla^{2}$. This simplification relies on first applying the Poisson operator $P$ to a Coulomb fitting basis, and then undoing its action to obtain the Coulomb potential. This is legitimate as the integration constants are fixed by the known asymptotic form of the potential, $v(\mathbf{r}) \sim Z / r$, where $Z$ is the total charge of the system.

Owing to the choice of the auxiliary basis we have

$$
\int d \mathbf{r} \tilde{\rho}(\mathbf{r})=0
$$

so we see that without further modification the method is applicable only to electrically neutral systems. In the context of quantum chemistry, this suggests that we may consider neutral molecules, providing we include both electrons and nuclei in the density. Since the nuclei are described by Dirac $\delta$ functions, we must be careful about our definition of integration [12], and put

$$
\int d \mathbf{r}_{1} \int d \mathbf{r}_{2} \frac{\delta\left(\mathbf{r}_{1}\right) \delta\left(\mathbf{r}_{2}\right)}{\left|\mathbf{r}_{1}-\mathbf{r}_{2}\right|} \stackrel{\text { def }}{=} 0 .
$$

This restriction, however, confers a great advantage: we effectively represent the Coulomb potential in the basis set $\left\{\xi_{a}\right\}$, and the short-ranged nature of the Coulomb potential for a neutral system will allow an accurate description without too large a basis. To illustrate this, the electronic, nuclear, and total Coulomb potentials for the hydrogen atom are shown in Fig. 1. The total potential is singular at the nuclei, so special basis functions are required to describe the singularity at short range but which vanish at long range. A suitable choice is

$$
f_{A}(\mathbf{r})=Z_{A} \frac{\operatorname{erfc}(\sqrt{\pi}|\mathbf{r}-\mathbf{A}| / 2)}{|\mathbf{r}-\mathbf{A}|}
$$

for a nucleus of charge $Z_{A}$ centered at $\mathbf{A}$. The coefficients of the $P f_{A}$ in the density are fixed at unity to give an exact description of the singularities. Then the rest of the basis $\left\{\xi_{a}\right\}$ is used to describe the sum of the electronic and long-ranged nuclear potentials. These potentials are illustrated for hydrogen in Fig. 1, where it will be seen that the quantity to be described by the remaining basis functions is extremely small, smooth, and short ranged, contributing less than $3 \%$ to the total Coulomb energy. By

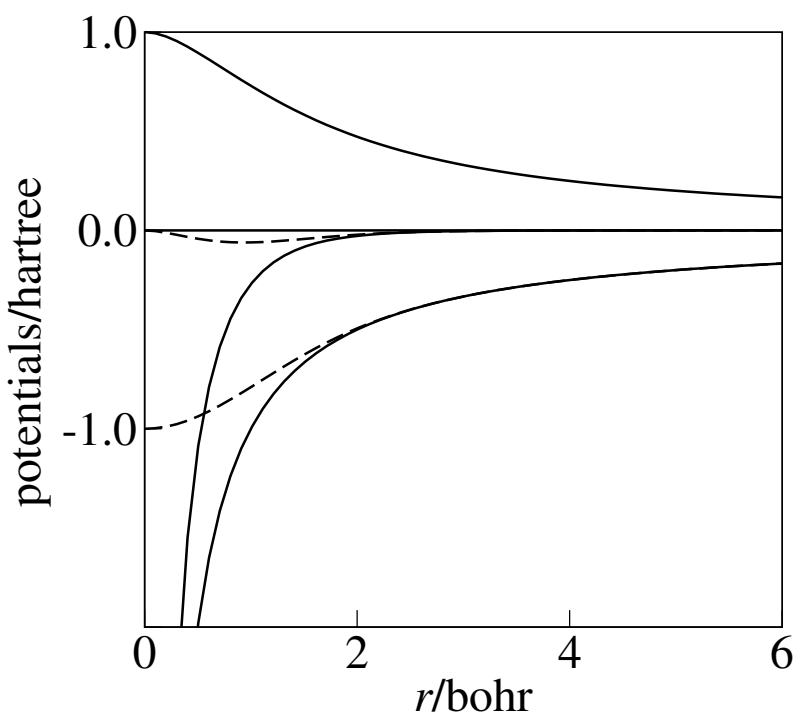

FIG. 1. Coulomb potentials for the hydrogen atom. Solid lines: electronic, nuclear, and total Coulomb potentials. Dashed lines: long-range nuclear potential $[\operatorname{erf}(\sqrt{\pi} r / 2) / r]$ and the sum of the electronic and long-range nuclear potentials.

performing the calculation with a single function of the form $\alpha r \exp \left(-\beta r^{2}\right)$, we obtain -0.687423 hartree for the total Coulomb energy of the hydrogen atom, comparing with the exact value of -0.6875 hartree.

To demonstrate the method for a molecule we optimized basis sets for hydrogen and fluorine by minimizing the error in Eq. (5) for, respectively, the $\mathrm{H}_{2}$ and $\mathrm{F}_{2}$ molecules at their equilibrium bond lengths using the cc-pVDZ orbital basis set [13]. The uncontracted even-tempered Gaussian basis sets are $12 s 6 p 4 d 1 f$ and $4 s 4 p 1 d$ for fluorine and hydrogen, respectively. Kohn-Sham calculations were then performed for the HF molecule using the local density approximation $[14,15]$ and the Coulomb contribution treated either exactly or with the current method. The total energy differs by 0.002 hartree, and the true and approximated dipole moments are, respectively, 0.7358 a.u. and 0.7363 a.u. The corresponding equilibrium bond lengths and harmonic stretching wave numbers are 1.767 and $1.761 \mathrm{bohr}$ and 3946 and $3965 \mathrm{~cm}^{-1}$. These errors are similar to those found in standard Coulomb fitting methods, and lend confidence to the hope that the proposed technique will offer comparable accuracy, but with very significantly reduced computational resources.

F.R.M. is grateful to the Royal Society for financial support.

*Present address: School of Chemistry, University of Bristol, Bristol BS8 1TS, United Kingdom.

${ }^{\dagger}$ To whom correspondence should be addressed.

[1] W. Kohn and L. J. Sham, Phys. Rev. 140, 1133 (1965).

[2] L. Greengard and V. Rokhlin, J. Comput. Phys. 73, 325 (1987). 
[3] L. Greengard, Science 265, 5174 (1994).

[4] C. A. White, B. G. Johnson, P. M. W. Gill, and M. HeadGordon, Chem. Phys. Lett. 230, 1 (1994).

[5] J. P. Dombroski, S. W. Taylor, and P. M. W. Gill, J. Phys. Chem. 100, 6272 (1996).

[6] M. C. Strain, G. E. Scuseria, and M. J. Frisch, Science 271, 5245 (1996).

[7] O. Vahtras, J. Almlöf, and M. W. Feyereisen, Chem. Phys. Lett. 213, 514 (1993).

[8] K. Eichkorn, O. Treutler, H. Öhm, M. Häser, and R. Ahlrichs, Chem. Phys. Lett. 240, 283 (1995).
[9] V. Termath and N. C. Handy, Chem. Phys. Lett. 230, 1 (1994).

[10] P. M. Morse and H. Feshbach, Methods of Theoretical Physics (McGraw-Hill, New York, 1953), Sec. 1.4.

[11] S. Obara and A. Saika, J. Chem. Phys. 84, 3963 (1986).

[12] P. M. W. Gill, Chem. Phys. Lett. 270, 193 (1997).

[13] T. H. Dunning, J. Chem. Phys. 90, 1007 (1989).

[14] P. A. M. Dirac, Proc. Cambridge Philos. Soc. 26, 376 (1930).

[15] S. J. Vosko, L. Wilk, and M. Nusair, Can. J. Phys. 58, 1200 (1980). 